\title{
A note on a family of multiplicative and additive mappings preserving the class $\operatorname{IFS}(X)$
}

\author{
Peter Vassilev \\ Institute of Biophysics and Biomedical Engineering \\ Bulgarian Academy of Sciences \\ 105 Acad. G. Bonchev Str., Sofia 1113, Bulgaria \\ e-mail: peter.vassilev@gmail.com
}

To the memory of Prof. Beloslav Riečan

Received: 15 September 2018 Revised: 29 October 2018 Accepted: 12 November 2018

\begin{abstract}
In the present paper we consider additive and multiplicative mappings which preserve the class of intuitionistic fuzzy sets defined over the same universe set $X$.
\end{abstract}

Keywords: Intuitionistic fuzzy set, Mapping, Preserving.

2010 Mathematics Subject Classification: 03E72.

\section{Introduction}

Our main motivation in trying to formally investigate the matter of additive and multiplicative preserving mappings regarding the class of intuitionistic fuzzy sets defined on the same universe set stems from the fact that many "generalizations" and "extensions" of the said sets may be reduced to some form of such mappings.

We will briefly remind some basic definitions and notions.

Let $X$ be a universe set, $A \subset X, \mu_{A}: X \rightarrow[0,1]$ and $\nu_{A}: X \rightarrow[0,1]$ are mappings reflecting the degree of membership and non-membership of the element $x \in X$ to the set $A$, respectively, such that for every $x$ it is fulfilled that

$$
\mu_{A}(x)+\nu_{A}(x) \leq 1
$$


Definition 1 (cf. [1]). We call the set

$$
A^{*} \stackrel{\text { def }}{=}\left\{\left\langle x, \mu_{A}(x), \nu_{A}(x)\right\rangle \mid x \in X\right\}
$$

an intuitionistic fuzzy set (IFS) and the mapping $\pi_{A}: X \rightarrow[0,1]$, which is given in explicit form by

$$
\pi_{A}(x) \stackrel{\text { def }}{=} 1-\mu_{A}(x)-\nu_{A}(x),
$$

is called degree of non-determinacy (uncertainty) (sometimes also: hesitancy margin or degree of indeterminacy) of the element $x$ (cf. [5]).

The class of all intuitionistic fuzzy sets over a universe set $X$ will be denoted further by $\operatorname{IFS}(X)$.

We will also further consider an equivalent multiplicative analogue of (1), namely:

$$
\mu_{A}(x) \nu_{A}(x) \leq\left(1-\mu_{A}(x)\right)\left(1-\nu_{A}(x)\right)
$$

Definition 2. We shall call a mapping $Z: A \rightarrow \operatorname{IFS}(X)$ an additive mapping iff

$$
Z(A)=\left\{\left\langle x, \mu_{A}(x)+\alpha_{\mu_{A}(x)}, \nu_{A}(x)+\beta_{\nu_{A}(x)}\right\rangle \mid x \in X\right\},
$$

with $\alpha_{\mu_{A}(x)}, \beta_{\nu_{A}(x)}$ - real valued constants. Obviously, it is assumed that:

$$
0 \leq \mu_{A}(x)+\alpha_{\mu_{A}(x)} \leq 1,0 \leq \nu_{A}(x)+\beta_{\nu_{A}(x)} \leq 1, \mu_{A}(x)+\alpha_{\mu_{A}(x)}+\nu_{A}(x)+\beta_{\nu_{A}(x)} \leq 1
$$

Remark 1. We need to mention that any such mapping $Z$ can be represented by the operator 0 , defined by (see [3]):

$$
\bigcirc_{\alpha, \beta, \gamma, \delta, \varepsilon, \zeta} A=\left\{\left\langle x, \alpha \mu_{A}(x)-\varepsilon \nu_{A}(x)+\gamma, \beta \nu_{A}(x)-\zeta \mu_{A}(x)+\delta\right\rangle \mid x \in X\right\},
$$

where $\alpha, \beta, \gamma, \delta, \varepsilon, \zeta \in[0,1]$ and

$$
0 \leq \min (\alpha-\zeta, \beta-\varepsilon)+\gamma+\delta \leq \max (\alpha-\zeta, \beta-\varepsilon)+\gamma+\delta \leq 1,
$$

when the above mentioned operator produces non-negative values for the membership and nonmembership functions. However, since this operator involves more than just additive mappings, we will not consider it further.

Definition 3. We shall call a mapping $Z: A \rightarrow \operatorname{IFS}(X)$ a multiplicative mapping iff

$$
Z(A)=\left\{\left\langle x, \alpha_{\mu_{A}(x)} \mu_{A}(x), \beta_{\nu_{A}(x)} \nu_{A}(x)\right\rangle \mid x \in X\right\},
$$

with $\alpha_{\mu_{A}(x)}, \beta_{\nu_{A}(x)} \geq 0$ - real valued constants. Obviously, it is assumed that:

$$
0 \leq \alpha_{\mu_{A}(x)} \mu_{A}(x) \leq 1,0 \leq \beta_{\nu_{A}(x)} \nu_{A}(x) \leq 1, \alpha_{\mu_{A}(x)} \mu_{A}(x)+\beta_{\nu_{A}(x)} \nu_{A}(x) \leq 1 .
$$

Remark 2. The above multiplicative mapping may be viewed as an extension of the operator $G_{\alpha, \beta}$ and its variant $G_{B}$ (see [3]) by values greater than 1 . Similar connection may be established between the additive mappings and the operator $F_{B}$ by considering addition of negative values. 
Further, we will also employ the concept of intuitionistic fuzzy pairs in our investigation.

Definition 4 (cf. [4]). An intuitionistic fuzzy pair (IFP) is an ordered couple of real non-negative numbers $\langle a, b\rangle$, with the additional constraint:

$$
a+b \leq 1 .
$$

Definition 5 (cf. [4]). Given two IFPs $u=\langle a, b\rangle$ and $v=\langle c, d\rangle$, they can be in the following distinct relations (There is a certain deviation from the original definitions given in [4] to make the cases disjoint):

$$
\begin{aligned}
& u<v \text { iff } a<c \text { and } b>d \\
& u>v \text { iff } a>c \text { and } b<d \\
& u=v \text { iff } a=c \text { and } b=d \\
& u \geqslant v \text { iff } \max (a, c)=a, \min (b, d)=b \text { and } a-c+d-b>0 \text { and }(a-c)(d-b)=0 \\
& u \leqslant v \text { iff } \min (a, c)=a, \max (b, d)=b \text { and } c-a+b-d>0 \text { and }(a-c)(d-b)=0 \\
& u \nsim v \text { otherwise }
\end{aligned}
$$

The last denotation means that the pairs are incomparable under the partial ordering.

\section{Describing the additive and multiplicative mappings}

In order to better understand how the additive mappings work, we will look at possible transformations with regard to a single point. In a sense, this may be viewed as manipulating an intuitionistic fuzzy pair labeled by the elements of the universe set $X$. Let $f$ be some type of additive or multiplicative admissible mapping. This means that $f$ transforms the intuitionistic fuzzy pair $u=\langle a, b\rangle$ to an intuitionistic fuzzy pair $v=\langle a, b\rangle$, i.e., we have: $f(u)=v$.

All the possible relations are given by Equations (7)-(12). In order to classify them properly, we will consider the mapping $f$ as a composition of two mappings $f_{L}$ and $f_{R}$-acting on the first and second component, respectively. Each of the two mappings can belong to three possible classes: identity (Id), strictly increasing $(\uparrow)$, or strictly decreasing $(\downarrow)$ mappings.

From Table 1 we see that for the cases where one of $f_{L}$ or $f_{R}$ coincides with Id, it is easy to establish the range of all admissible mappings, as is the case when $\left(f_{L}, f_{R}\right) \in\{\downarrow, \downarrow\}$.

\begin{tabular}{|c|c|}
\hline Relation of $u, v$ & $\left(f_{L}, f_{R}\right) \in$ \\
\hline$(7)$ & $\{\uparrow, \downarrow\}$ \\
\hline$(8)$ & $\{\downarrow, \uparrow\}$ \\
\hline$(9)$ & $\{\mathrm{Id}, \mathrm{Id}\}$ \\
\hline$(10)$ & $\{\mathrm{Id}, \downarrow\},\{\uparrow, \mathrm{Id}\}$ \\
\hline$(11)$ & $\{\mathrm{Id}, \uparrow\},\{\downarrow, \mathrm{Id}\}$ \\
\hline$(12)$ & $\{\uparrow, \uparrow\},\{\downarrow, \downarrow\}$ \\
\hline
\end{tabular}

Table 1. Possible admissible mappings 
In particular, if $f$ is the identity mapping in terms of additive mappings, this is equivalent to addition of zero. If it is multiplicative, this is equivalent to multiplication by 1 .

When $\left(f_{L}, f_{R}\right) \in\{\downarrow, \downarrow\}$, this corresponds to multiplicative mappings with constants in the interval $[0,1)$. In the additive case, this corresponds to subtraction of non-negative constants which are not greater than the current values of the IFP.

The possible cases that remain are when

$$
\left(f_{L}, f_{R}\right) \in\{\downarrow, \uparrow\} \cup\{\uparrow, \downarrow\} \cup\{\uparrow, \uparrow\}
$$

Let us first consider the case $\left(f_{L}, f_{R}\right) \in\{\downarrow, \uparrow\}$, hence $u>v$, i.e.,

$$
\left\{\begin{array}{l}
a>f_{L}(a)=c \\
b<f_{R}(b)=d
\end{array} .\right.
$$

If $1 \geq a+b \geq f_{L}(a)+f_{R}(b)$, the mapping is obviously admissible. This may be rewritten as: $a-f_{L}(a) \geq f_{R}(b)-b$.

In the case of additive mapping: $a \geq \varepsilon_{a}^{-} \geq \varepsilon_{b}^{+}$, where $\varepsilon_{a}^{-}$is the quantity subtracted from $a$ and $\varepsilon_{b}^{+}$is the quantity added to $b$. In the case of multiplicative mapping: $\left(1-\alpha_{\downarrow}\right) a \geq\left(\beta_{\uparrow}-1\right) b$. Assuming, $a b \neq 0$, this yields

$$
\left\{\begin{array}{l}
0 \leq \alpha_{\downarrow} \leq 1 \\
\beta_{\uparrow} \leq \frac{a+b-\alpha_{\downarrow} a}{b}
\end{array}\right.
$$

Let now $a+b<f_{L}(a)+f_{R}(b)$, then an admissible mapping should satisfy

$$
\left\{\begin{array}{l}
\varepsilon_{a}^{-}=a-f_{L}(a)<f_{R}(b)-b=\varepsilon_{b}^{+} \\
f_{L}(a) \leq 1-f_{R}(b)
\end{array} .\right.
$$

The last is true for additive mappings when

$$
\left\{\begin{array}{l}
\varepsilon_{a}^{-} \leq a \\
\varepsilon_{b}^{+} \leq 1-b \\
\varepsilon_{b}^{+}-\varepsilon_{a}^{-} \leq 1-b-a
\end{array},\right.
$$

and for multiplicative mappings (assuming $a b \neq 0$ ) when

$$
\left\{\begin{array}{l}
0<\alpha_{\downarrow}<1 \\
\beta_{\uparrow} \leq \frac{1-\alpha_{\downarrow} a}{b}
\end{array}\right.
$$

Let us now consider the case $\left(f_{L}, f_{R}\right) \in\{\uparrow, \downarrow\}$, hence $u<v$, i.e.,

$$
\left\{\begin{array}{l}
a<f_{L}(a)=c \\
b>f_{R}(b)=d
\end{array} .\right.
$$

If $1 \geq a+b \geq f_{L}(a)+f_{R}(b)$, the mapping is obviously admissible. This may be rewritten as: $\varepsilon_{b}^{-}=b-f_{R}(b) \geq f_{L}(a)-a=\varepsilon_{a}^{+}$. 
In the case of additive mapping: $b \geq \varepsilon_{b}^{-} \geq \varepsilon_{a}^{+}$, where $\varepsilon_{a}^{+}$is the quantity added to $a$ and $\varepsilon_{b}^{-}$ is the quantity subtracted from $b$. In the case of multiplicative mapping: $\left(1-\beta_{\downarrow}\right) b \geq\left(\alpha_{\uparrow}-1\right) a$. Assuming, $a b \neq 0$, this yields

$$
\left\{\begin{array}{l}
0 \leq \beta_{\downarrow}<1 \\
\alpha_{\uparrow} \leq \frac{a+b-\beta_{\downarrow} b}{a}
\end{array} .\right.
$$

Let now $a+b<f_{L}(a)+f_{R}(b)$, then an admissible mapping should satisfy

$$
\left\{\begin{array}{l}
\varepsilon_{b}^{-}=b-f_{R}(b)<f_{L}(a)-a=\varepsilon_{a}^{+} \\
f_{L}(a) \leq 1-f_{R}(b)
\end{array} .\right.
$$

The last is true for additive mappings when

$$
\left\{\begin{array}{l}
\varepsilon_{a}^{+} \leq 1-a \\
\varepsilon_{b}^{-} \leq b \\
\varepsilon_{a}^{+}-\varepsilon_{b}^{-} \leq 1-a-b
\end{array}\right.
$$

and for multiplicative mappings (assuming $a b \neq 0$ ) when

$$
\left\{\begin{array}{l}
0<\beta_{\downarrow}<1 \\
\alpha_{\uparrow} \leq \frac{1-\beta_{\downarrow} b}{a}
\end{array}\right.
$$

Finally, let us first consider the case $\left(f_{L}, f_{R}\right) \in\{\uparrow, \uparrow\}$, hence $u \nsim v$, and in particular:

$$
\left\{\begin{array}{l}
a<f_{L}(a)=c \\
b<f_{R}(b)=d
\end{array} .\right.
$$

Hence, an admissible mapping should satisfy:

$$
f_{L}(a) \leq 1-f_{R}(b)
$$

For additive mappings, this is equivalent to:

$$
\varepsilon_{a}^{+}+\varepsilon_{b}^{+} \leq 1-a-b,
$$

where $f_{L}(a)-a=\varepsilon_{a}^{+}$and $\varepsilon_{b}^{+}=f_{R}(b)-b$, respectively.

For multiplicative mappings (assuming $a b \neq 0$ ) we should have:

$$
\left\{\begin{array}{l}
1<\alpha_{\uparrow}<\frac{1-b}{a} \\
\beta_{\uparrow} \leq \frac{1-\alpha_{\uparrow} a}{b}
\end{array} .\right.
$$

We will further present the tables with all admissible mappings and relevant constraints (Tables 2 and 3). Since we can treat the identity mapping as $\uparrow$ with $\varepsilon^{+}=0$, or as $\downarrow$ with $\varepsilon^{-}=0$, for additive mappings and as $\uparrow$ with $\delta_{\uparrow}=1$, or as $\downarrow$ with $\delta_{\downarrow}=1$, where $\delta \in\{\alpha, \beta\}$, these are omitted in the tables. 


\begin{tabular}{|c|c|c|}
\hline Initial IFP $(a, b)$ & Mappings type & Constraints \\
\hline \multirow{5}{*}{$(a, b)$} & $\{\uparrow, \downarrow\}$ & $\left\{\begin{array}{l}\varepsilon_{a}^{+}-\varepsilon_{b}^{-} \leq 1-a-b \\
\varepsilon_{a}^{+} \leq 1-a \\
\varepsilon_{b}^{-} \leq b\end{array}\right.$ \\
\cline { 2 - 3 } & $\{\downarrow, \uparrow\}$ & $\left\{\begin{array}{l}\varepsilon_{b}^{+}-\varepsilon_{a}^{-} \leq 1-a-b \\
\varepsilon_{b}^{+} \leq 1-b \\
\varepsilon_{a}^{-} \leq a\end{array}\right.$ \\
\cline { 2 - 3 } & $\{\downarrow, \downarrow\}$ & $\left\{\begin{array}{l}\varepsilon_{a}^{-} \leq a \\
\varepsilon_{b}^{-} \leq b\end{array}\right.$ \\
\cline { 2 - 3 } & $\{\uparrow, \uparrow\}$ & $\left\{\begin{array}{l}\varepsilon_{a}^{+} \leq 1-a \\
\varepsilon_{b}^{+} \leq 1-b \\
\varepsilon_{a}^{+}+\varepsilon_{b}^{+} \leq 1-a-b\end{array}\right.$ \\
\hline
\end{tabular}

Table 2. Possible admissible additive mappings

\begin{tabular}{|c|c|c|}
\hline Initial IFP $(a, b)$ & Mappings type & Constraints \\
\hline \multirow{4}{*}{$(a, b)$} & $\{\uparrow, \downarrow\}$ & $\begin{array}{l}\alpha_{\uparrow} \leq \frac{1-\beta_{\downarrow} b}{a} \\
0<\beta_{\downarrow}<1\end{array}$ \\
\hline & $\{\downarrow, \uparrow\}$ & $\begin{array}{l}\alpha_{\downarrow}<1 \\
0<\beta_{\uparrow}<\frac{1-\alpha_{\downarrow} a}{b}\end{array}$ \\
\hline & $\{\downarrow, \downarrow\}$ & $\begin{array}{l}0<\alpha<1 \\
0<\beta<1\end{array}$ \\
\hline & $\{\uparrow, \uparrow\}$ & $\left\{\begin{array}{l}1<\alpha_{\uparrow}<\frac{1-b}{a} \\
\beta_{\uparrow} \leq \frac{1-\alpha_{\uparrow} a}{b}\end{array}\right.$ \\
\hline
\end{tabular}

Table 3. Possible admissible multiplicative mappings $(a b \neq 0)$.

Finally, we will make some comments regarding the possible representation of the said mappings via the use of intuitionistic fuzzy sets and we will provide some additional results.

Bearing in mind (4), all additive mappings must result in non-negative pairs and the sum of their components should be less or equal to 1 . Thus, returning to IFS interpretation, we can conclude that for any particular $x$, the following should hold

$$
\alpha_{\mu_{A}(x)}=\alpha_{A}(x)^{*}-\mu_{A}(x), \beta_{\nu_{A}(x)}=\beta_{A}(x)^{*}-\nu_{A}(x),
$$

with $\alpha_{A}(x)^{*} \geq 0, \beta_{A}(x)^{*} \geq 0$ and $\alpha_{A}(x)^{*}+\beta_{A}(x)^{*} \leq 1$. 
Therefore, any additive mapping can be represented as a function of two intuitionistic fuzzy sets in the following manner:

$$
f(A, B)=\left\{\langle x, \mu_{A}(x)+\underbrace{\alpha_{\mu_{A, B}(x)}}_{=\mu_{B}(x)-\mu_{A}(x)}, \nu_{A}(x)+\underbrace{\beta_{\nu_{A, B}(x)}}_{=\nu_{B}(x)-\nu_{A}(x)}\rangle \mid x \in X\right\}=B .
$$

for an appropriately chosen intuitionistic fuzzy set $B$.

In the case of multiplicative mappings we cannot easily represent it with the help of another intuitionistic fuzzy set. However, for $\{\uparrow, \uparrow\}$ (or $\{\uparrow, \operatorname{Id}\},\{\operatorname{Id}, \uparrow\}$ ) we can give the following bound from above for $\min \left(\alpha_{\uparrow}, \beta_{\uparrow}\right)$ given an initial IFP $(a, b)$,

$$
\min \left(\alpha_{\uparrow}, \beta_{\uparrow}\right) \leq \frac{1}{a+b}
$$

\section{Conclusion}

We have given a partial characterization of the multiplicative and additive mappings and have summarized the required constraints. In the near future, we plan to investigate different possibilities for extending the concept of such mappings to the case of interval valued intuitionistic fuzzy sets.

\section{Acknowledgements}

Work presented here is supported by Program for Career Development of Young Scientists, Bulgarian Academy of Sciences, Grant number DFNP-17-139 "Investigation of different extensions of fuzzy sets and assessment of their correctness".

\section{References}

[1] Atanassov, K. (1983). Intuitionistic fuzzy sets. VII ITKR Session, Sofia 20-23 June, 1983 (Central Sci. and Techn. Library, Bulg. Academy of Sciences, 1984). (in Bulgarian). Reprinted: Int. J. Bioautomation, 2016, 20(S1), S1-S6 (in English).

[2] Atanassov, K. (1989). Intuitionistic fuzzy sets, Fuzzy Sets and Systems, 2, 87-96.

[3] Atanassov, K. (2012). On Intuitionistic Fuzzy Sets Theory, Springer-Verlag Berlin Heidelberg.

[4] Atanassov, K., Szmidt, E., \& Kacprzyk, J. (2013). On intuitionistic fuzzy pairs, Notes on Intuitionistic Fuzzy Sets, 19(3), 1-13.

[5] Szmidt, E. (2014). Distances and Similarities in Intuitionistic Fuzzy Sets. Springer, Heidelberg. 